
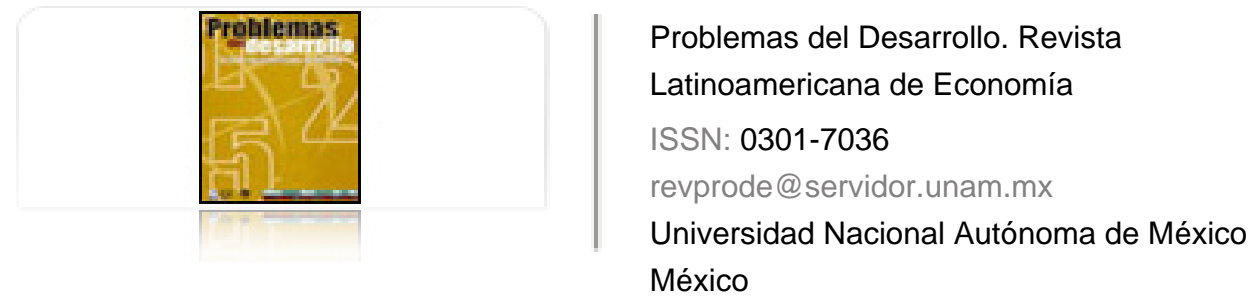

Sánchez Almanza, Adolfo

Crecimiento económico, desigualdad y pobreza: una reflexión a partir de Kuznets Problemas del Desarrollo. Revista Latinoamericana de Economía, vol. 37, núm. 145, abril-junio, 2006, pp. 11-30

Universidad Nacional Autónoma de México

Distrito Federal, México

Disponible en: http://www.redalyc.org/articulo.oa?id=11820086002

Cómo citar el artículo

Número completo

- Más información del artículo

Página de la revista en redalyc.org

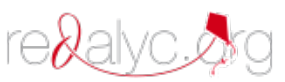

Sistema de Información Científica

Red de Revistas Científicas de América Latina, el Caribe, España y Portugal Proyecto académico sin fines de lucro, desarrollado bajo la iniciativa de acceso abierto 


\section{CRECIMIENTO ECONÓMICO, DESIGUALDAD Y POBREZA: UNA REFLEXIÓN A PARTIR DE KUZNETS}

\section{Adolfo Sánchez Almanza*}

Fecha de recepción: 16 de diciembre de 2005. Fecha de aceptación: 27 de abril de 2006.

\section{Resumen}

En este trabajo se exponen algunas reflexiones teóricas básicas a partir de la hipótesis de Kuznets que relaciona el crecimiento económico con cambios en la desigualdad en el ingreso de la población, se revisan algunas aportaciones de otros autores en esa línea y se analiza el comportamiento de esas variables en América Latina y México mediante datos representativos en series de largo plazo; asimismo se concluye con una breve lista de propuestas relacionadas con mecanismos redistributivos dirigidos a la población más pobre.

Palabras clave: crecimiento económico, desigualdad, ingreso, población y mecanismos redistributivos.

\section{Abstract}

This study makes some basic theoretical reflections using the Kuznets hypothesis which relates economic growth to changes in the population's income inequality, reviewing some of the contributions of other authors in this area and analyzing the performance of these variables in Latin America and Mexico via representative data in long-term series; it also concludes with a brief list of proposals related to redistributive mechanisms targeting the poorest part of the population.

Key words: economic growth, inequality, income, population and redistributive mechanisms.

* Investigador Asociado en el Instituto de Investigaciones Económicas de la UNAM donde es Coordinador del Seminario de Economía Urbana y Regional. Correo electrónico: asag@servidor.unam.mx 


\section{Résumé}

Dans ce travail on expose quelques réflexions théoriques de base à partir de l'hypothèse de Kuznets qui met en relation la croissance économique avec des changements dans l'inégalité du revenu de la population, on y révise certains apports d'autres auteurs dans cette même ligne et on analyse le comportement de ces variables en Amérique Latine et au Mexique au moyen de données représentatives dans des séries à long terme; en conclusion, une courte liste de propositions en relation avec des mécanismes redistributifs dirigés vers la population la plus pauvre.

Mots-cléfs: croissance économique, inégalité, revenu, population et mécanismes redistributifs.

\section{Resumo}

Neste trabalho apresentam-se algumas reflexões teóricas básicas a partir da hipótese de Kuznets que relaciona o crescimento econômico com mudanças na desigualdade no ingresso da população, revisam-se algumas aportações de outros autores nessa linha e se analiza o comportamento destas variáveis Na América Latina e no México mediante dados representativos em séries de longo prazo; igualmente, se conclui com uma concisa listagem das propostas relacionadas aos mecanismos redistributivos dirigidos à população mais pobre.

Palavras chave: Crescimento econômico, desigualdade, ingresso, população e mecanismos redistributivos. 


\section{Introducción}

$\mathrm{L}$

a desigualdad y la pobreza son dos de los más graves problemas sociales que, en general, resultan de las relaciones históricas que se establecen entre las esferas de

la sociedad, el Estado y el mercado. Esos fenómenos se observan a escala mundial entre regiones y países, y al interior de cada uno de éstos. En una línea de investigación se los ha relacionado con algunas dimensiones como el crecimiento económico, la distribución del ingreso, el comportamiento del gasto público y las condiciones sociales analizadas mediante diferentes variables e indicadores. En este trabajo se revisan algunas propuestas teóricas y análisis empíricos asociados a los planteamientos formulados por Simón Kuznets y a su aplicación a los casos de América Latina y México.

\section{Modelos de distribución}

La relación entre crecimiento económico y distribución del ingreso ha sido estudiada por autores de diversas corrientes teóricas (véase Aguilera, 1998). Sin embargo, hasta la década de los setenta predominó la propuesta de Simón Kuznets, según la cual el reto económico central era lograr el crecimiento económico por medio de la modernización del aparato productivo, lo que a su vez permitiría reducir la desigualdad en la distribución del ingreso. Esta hipótesis era consistente con la experiencia de la mayoría de los países desarrollados. En naciones atrasadas este proceso sería una curva en forma de $\mathrm{U}$ invertida. En una primera fase A de crecimiento económico aumentaría la desigualdad en la distribución del ingreso, pero habría transferencias de mano de obra de sectores rurales y agrícolas de baja productividad hacia sectores urbanos e industriales de alta productividad en una fase $\mathrm{B}$, con lo cual se ampliaría el mercado a los productos primarios, la desigualdad se estabilizaría por un tiempo, y luego se reduciría. Dicha hipótesis supone: $a$ ) un dualismo sectorial con diferencias de productividad en la economía, $b$ ) una elevada proporción de la población dependiente de actividades agrícolas, $c$ ) libre transferencia de población hacia el sector no agrícola acorde con el proceso de crecimiento económico y $d$ ) la productividad, la distribución del ingreso y la desigualdad en el sector no agrícola mayores que en el agrícola. Con estos supuestos los cambios en la desigualdad dependen de: 1) las diferencias de desigualdad entre sectores, 2) la concentración de la propiedad y los activos (ahorro) y 3) las políticas redistributivas que son aplicadas en las etapas avanzadas de crecimiento (Gráfica 1). ${ }^{1}$

Kuznets, en las conclusiones de su trabajo pionero, acepta que tal vez $5 \%$ sea información empírica y $95 \%$ especulación, y que en alguna de ellas posiblemente exista una contaminación de ilusiones

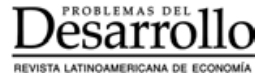




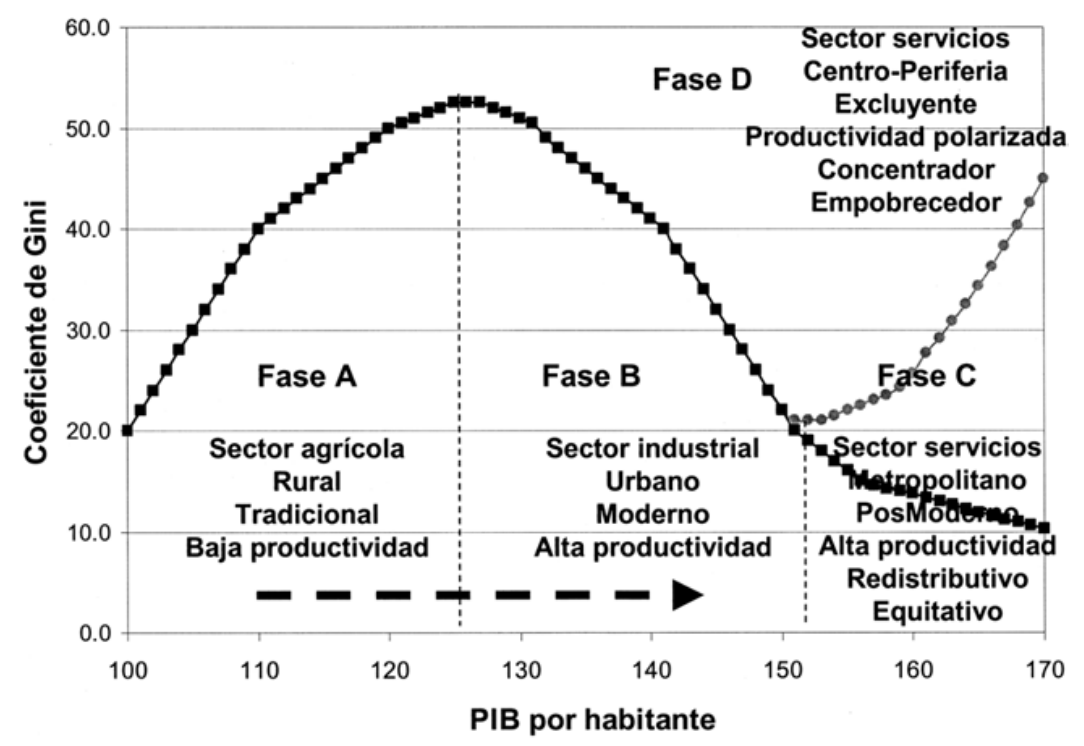

Gráfica 1. Curva de Kuznets y nuevas tendencias.

La hipótesis de Kuznets la confirman otros autores como Arthur Lewis, quien desarrolló un modelo teórico en el cual plantea que el crecimiento y la acumulación de capital se darían en el sector industrial moderno, los capitalistas contratarían un salario determinado y reinvertirían parte de sus ganancias; la distribución del ingreso mejoraría con la transferencia de trabajadores del sector tradicional de baja productividad al moderno; la desigualdad en el ingreso aumentaría inicialmente como promedio del alza del ingreso mismo, pero después se reduciría al pasar de una economía dual a una economía de un solo sector totalmente industrializado. Ese proceso supone un alto ritmo de crecimiento. Por su parte, Lydall desarrolla un modelo de dos sectores: el tradicional y el moderno, con variaciones en la productividad y la distribución intrasectorial del ingreso. A su vez, Robinson demuestra que existe un punto en el crecimiento económico en el cual la desigualdad alcanza su valor máximo y luego disminuye.

Sin embargo, otros investigadores cuestionan el modelo. Kravis, por ejemplo, señala que en la fase de industrialización el excedente y la dispersión de los ingresos aumentan rápidamente, y con ello la desigualdad, pero después no se puede determinar una tendencia clara. Bourguignon establece que la distribución en el ingreso debe tomar en cuenta la

(Kuznets, 1955). De igual manera, en otro trabajo hace una observación metodológica: "para asegurar una perspectiva adecuada y distinguir los movimientos a largo plazo de las fluctuaciones a corto plazo que acompañan a los ciclos económicos o a otros cambios pasajeros necesitamos estimaciones que cubran un periodo suficientemente largo. En mi propio trabajo [...] utilizo una extensión de cuatro o cinco décadas como periodo mínimo [...] en los Estados Unidos, los tipos de crecimiento revelan oscilaciones de 20 a 25 años, además de las fluctuaciones cíclicas que varían de cuatro a nueve años" (Kuznets, 1963:7).

\section{DeSarrollo}


magnitud de las elasticidades precio e ingreso en cada sector y las fases en que se encuentre la economía; asimismo, apunta el papel relevante de la tecnología utilizada, que generalmente amplía la brecha intersectorial. Más recientemente, otros autores como Deininger y Squire demostraron que la curva de Kuznets no es sólida si se consideran bases de datos más amplias, diferente composición en la muestra de países utilizada y varios periodos de observación (Hernández, 1999). La relación causal entre crecimiento y desigualdad ha sido reexaminada y se han analizado sus relaciones con otras variables. Por ejemplo, Galor y Zeira estudian la contracción de los préstamos que disminuye la inversión en capital humano, lo que afecta la distribución del ingreso y reduce el producto en el largo plazo; Aghión y Bolton exploran las imperfecciones de los mercados de capital; Alesina, Rodrik y Perroti investigaron las implicaciones de la distribución del ingreso, mediante procesos políticos, en el crecimiento económico; Benabou y Durlauf la examinaron en función del patrón de asentamientos humanos; y Galor y Zang analizaron el papel de la fecundidad y la distribución del ingreso en el crecimiento del producto (Galor y Tsiddon, 1996).

En un estudio que confirma la hipótesis de Kuznets se elabora un modelo de equilibrio general, según el cual el crecimiento del producto es acompañado en las fases iniciales de desarrollo por una ampliación en la brecha entre los salarios de trabajadores calificados y los no capacitados, mientras que en el largo plazo esta diferencia disminuye. Más aún se afirma que, en esas etapas iniciales del desarrollo, un incremento en el nivel agregado de inversión en capital humano puede no ser viable, a menos que la distribución de éste (y en consecuencia la distribución del ingreso) sea desigual, aunque no señalan por cuánto tiempo y aplican varios supuestos insostenibles tal como una competencia mundial perfecta (ibid.).

En otro trabajo clásico, Bruno, Ravallion y Squire, al analizar datos de 44 países por década entre 1960 y 1990, no encontraron evidencias de la curva invertida, y al profundizar en el caso de la India tampoco encontraron que el crecimiento económico aumente la desigualdad. En su trabajo señalan que no se puede afirmar que el crecimiento siempre beneficie a los pobres o que la pobreza no se reduce por efecto de políticas pro crecimiento. La relación entre ambas es compleja y no presenta tendencias claras generales. Hay otros factores que influyen en la desigualdad, por ejemplo, la educación, el comercio o la composición sectorial del crecimiento. Asimismo, concluyen que las políticas dirigidas a los pobres mediante las cuales se acumulan activos productivos — principalmente en educación, salud y nutrición - cuando son aplicadas en un contexto "sin distorsiones", son instrumentos importantes para lograr un alto crecimiento (Bruno, Ravallion y Squire, 1996).

Otros estudios indican que la relación entre crecimiento económico y desigualdad debe analizarse con más cuidado en los países subdesarrollados como los de América Latina, incorporando otras variables explicativas como: $a$ ) las de tipo histórico, político y cultural; b) considerar que la desigualdad es una función de la dependencia y parte de un sistema cultural y político corporativo, burocrático y autoritario, y c) tomar en cuenta la educación 
y las disparidades regionales en espacios subnacionales. Asimismo, es muy importante estudiar la propiedad de la tierra, ya que algunas naciones atrasadas con PIB relativamente alto y bajos niveles de desigualdad lo lograron debido a: 1) redistribución antes del crecimiento; por ejemplo, con la aplicación de políticas de distribución masiva de tierras en las etapas iniciales de desarrollo (como sucedió en México durante el gobierno de Lázaro Cárdenas); o 2) redistribución con crecimiento, con políticas redistributivas durante las fases de industrialización (como en la etapa de sustitución de importaciones de México con una fuerte política social, aunque de universalismo segmentado), y no necesariamente por una tendencia asociada con la curva de Kuznets (Bowman, 1997).

Como se puede ver, existe una amplia gama de líneas de investigación a partir de la propuesta seminal de Kuznets, la cual no se puede considerar una ley, sino una hipótesis que ha de verificarse en cada país en particular y en periodos específicos. No obstante, a partir de los modelos iniciales de inspiración neoclásica de los años cincuenta, se derivaron estrategias prácticas establecidas en los planes de desarrollo económico orientadas a fortalecer el proceso de industrialización y urbanización, con lo cual se incrementaría la productividad marginal de los factores, en el supuesto de la movilidad de la mano de obra del campo a las ciudades y su inserción al mercado laboral. A su vez, el reforzamiento de nodos urbanos dinámicos modernos, industrializados y con aplicación de innovaciones tecnológicas propiciaría la difusión del crecimiento hacia sus áreas de influencia, la mayoría tradicionales, atrasadas, rurales y pobres. Las políticas públicas se orientaron entonces a la creación y atracción de industrias mediante incentivos a la inversión y provisión de infraestructura para centros urbanos que atraían población. En consecuencia, se adoptaron estrategias de desarrollo sectorial desigual y de polarización territorial.

En la década de los setenta esos modelos estaban en crisis. A partir de los años ochenta se enfrentaron a una nueva realidad al producirse una recesión mundial en la cual gran número de economías tradicionales atrasadas redujeron bruscamente su crecimiento; se enfrentaron a un insuficiente desarrollo industrial que no pudo sostener el dinamismo económico, a modificaciones en los precios relativos asociados a choques externos y a la aplicación de medidas de estabilización y ajuste estructural. En ese contexto el sector moderno redujo su dinamismo y no fue capaz de asimilar a la población excedente originaria del sector tradicional, que tampoco mejoró su productividad por una baja tasa de inversión y la limitada incorporación de tecnología, pero que se refugió en actividades terciarias en condiciones precarias y en espacios periféricos metropolitanos con limitados servicios públicos. La consecuencia fue un aumento en la desigualdad socioespacial e intersectorial, sobre todo por el repunte en la concentración del ingreso, la caída del empleo formal, la contención del salario real directo como principal variable para controlar la inflación y el proceso general de empobrecimiento en muchas regiones, asociadas a una retracción del Estado como agente redistribuidor o compensador social en fases de crisis.

\section{DeSarrollo}


Es importante considerar en las últimas décadas fenómenos asociados con la mundialización del capitalismo que incluyen la movilidad del capital, la dislocación del proceso productivo, cambios en la división internacional del trabajo y mayor desarrollo del sector servicios apoyado en los avances científico-tecnológicos.

Este conjunto de tendencias corresponden a dos patrones, los cuales, siguiendo el razonamiento de Kuznets, permiten sugerir una fase $\mathrm{D}$, que aumenta la desigualdad o se mantiene en niveles altos (Gini ${ }^{2}$ entre 0.4 y 0.6 ) asociada con un modelo polarizado, excluyente, concentrador y empobrecedor con estancamiento económico en muchas naciones atrasadas; y en el otro extremo, una fase $\mathrm{C}$, con una tendencia asociada al modelo kusneztsiano, correspondiente sobre todo a países desarrollados y algunos asiáticos con un modelo posmoderno, de alta productividad, redistributivo y más equitativo (véase Gráfica 1).

Por otra parte, se afirma que la hipótesis de Kuznets, consistente en que la desigualdad en el ingreso se incrementa al principio con el crecimiento económico, pero después disminuye para lograr sociedades más desarrolladas, fue usada (sobre todo de manera ideológica en el Consenso de Washington desde una visión etapista) para justificar el crecimiento de la desigualdad en los países pobres y ampliar la brecha en el desarrollo internacional. Ante ello es necesario pasar del objetivo estratégico de "crecer primero" al de "crecer con equidad", sobre la base de las necesidades humanas con inclusión social (Mkandawire, 2001). Aún desde la economía neoclásica se avanza gradualmente del análisis de la relación causal entre crecimiento y distribución a la aceptación de los efectos positivos de mayores grados de equidad sobre un crecimiento más dinámico.

En ese contexto se incluyeron, en algunas estrategias de desarrollo nacional, políticas que integraban el crecimiento del producto con la equidad en el ingreso, por lo menos en tres variantes: a) el modelo chino, que enfatizó la distribución de la riqueza hacia zonas rurales mediante la expropiación de tierras y empresas para repartirlas entre pequeños productores y empresas estatales colectivas, con el objetivo de aumentar la productividad, el empleo y el ingreso, lo cual fortaleció las estrategias de desarrollo rural y endógeno, y permitió integrar los sectores tradicional y moderno; $b$ ) el modelo de la onU, orientado a un alto crecimiento económico cuyas ganancias se debían redistribuir mediante intervenciones gubernamentales tales como: provisión de bienes y servicios a los más necesitados, educación y capacitación para los trabajadores de bajos ingresos, y fomento a la generación y acumulación de activos de los pobres (mejoramiento de tierras e instrumentos de trabajo); y c) el modelo de necesidades básicas, que propuso una política de ataque a la pobreza mediante la provisión de bienes y servicios en aspectos de nutrición, salud, educación, vivienda y servicios básicos. En aportaciones subsecuentes, se reconsideró el papel de la agricultura en el desarrollo, sobre todo con el impulso a la agroindustria, la creación de infraestructura y la ampliación de servicios rurales que facilitaran la integración

2 Sobre el coeficiente de Gini véase Cortés y Ruvalcaba (1984). 
campo-ciudad, a la vez que se introdujeron los enfoques de desarrollo rural integral, de tipo endógeno y con sustentabilidad ambiental (Asuad, 2004).

Las bases conceptuales del análisis y la medición de la riqueza también se han reorientado hacia el desarrollo sustentable, entendido como aquel que "satisface las necesidades actuales sin comprometer la posibilidad de las generaciones futuras de cumplir con sus propios requerimientos", como lo definió la Comisión Brundtland en 1987. Con esta idea, la riqueza de un país incluye el capital físico (bienes manufacturados, edificios, maquinaria, carreteras, entre otros), pero también el capital humano (salud, conocimiento y capacidades), el capital natural (ecosistemas, minerales y combustibles fósiles) y las instituciones (gobierno, sociedad civil y estado de derecho). El desarrollo será sustentable si, y sólo si, la riqueza de una economía con relación a su población se mantiene en el tiempo. El crecimiento económico entonces forma parte de la riqueza generada, que no se debe entender sólo como incremento del producto interno bruto. A partir de ese nuevo paradigma se estima que durante las tres décadas pasadas, el mundo rico disfrutó de un "desarrollo sustentable", mientras que en el pobre (con excepción de China) no lo ha sido, en este caso porque aún cuando hubiera incrementado su PIB por habitante (con capital físico y capital humano) su riqueza habría disminuido, pues éste no ha compensado la degradación del capital natural, que además tiene límites de reposición como lo demuestra, entre otros fenómenos, el calentamiento global (Dasgupta, 2005).

El concepto de equidad tiene características multidimensionales; es decir, desde la dimensión económica el ingreso se complementa en lo social con variables de necesidades básicas, pero deben incluirse también otras dimensiones como la intergeneracional, la étnica, la de género y la territorial, las cuales expresan formas específicas de desigualdad.

Las reflexiones anteriores se elaboran a partir de la propuesta de Kuznets, la cual intentó explicar las relaciones entre crecimiento y desarrollo, así como formular políticas relacionadas a éstos. Análisis posteriores como $\operatorname{los} \operatorname{citados}^{3}$ han demostrado que no se trata de una ley ni un modelo único y que es necesario incorporar otros elementos para explicar su evolución en cada país y buscar estrategias alternativas más integrales, sobre todo adaptadas a realidades concretas de países subdesarrollados en el marco de una realidad hegemónica mundial.

\section{América Latina}

En las últimas dos décadas ha predominado una tendencia en América Latina: las fases de mayor crecimiento económico están fuertemente asociadas con mayor desigualdad en la posesión de la riqueza. Las condiciones iniciales de inequidad significan que una creciente

3 El Banco Mundial, citando la exhaustiva revisión de la literatura sobre la curva de Kuznets por Kanbur (2000), señala que hay cierto consenso en que no existe una relación clara entre el ingreso y la desigualdad (wB, 2005).

\section{DeSarrollo}


y gran cantidad de personas queda excluida de los beneficios del desarrollo. Ello se critica desde posiciones éticas, morales y de derechos humanos que consideran el bienestar social como un fin en sí mismo, garantizado por marcos institucionales y pactos internacionales; al tiempo que se acepta cada vez más la importancia de desarrollar el capital social y humano, superar la pobreza y mejorar la distribución social de la riqueza como requisito para potenciar el crecimiento económico desde el enfoque del desarrollo sustentable.

América Latina, de acuerdo con la tendencia mundial, se consolidó como la región más desigual en el planeta; la pobreza aumentó de manera sostenida en términos absolutos, aunque se llegue a estabilizar en términos relativos. A su vez, el crecimiento económico ha sido irregular y débil. Si bien debería producir una reducción relativa en la pobreza, en fases recesivas ésta crece $1.8 \%$ por cada punto en que disminuye el PIB por habitante, mientras que en fases de crecimiento sólo declina 0.6\% (CEPAL, 2001).

La desigualdad y la pobreza han formado parte de políticas y programas en el discurso oficial. Sin embargo, sus fracasos o limitantes se hacen evidentes con los resultados netos. En la búsqueda de un consenso alternativo, esos fenómenos se explican con varias argumentaciones que han evolucionado en el tiempo, entre las cuales se mencionan dos.

La primera, la teoría del derrame suponía que la pobreza se iba a superar logrando al principio mayor crecimiento económico. A pesar de que resultó insuficiente, en la práctica logró desplazar otras políticas más efectivas para mejorar la igualdad y abatir la pobreza. En América Latina se observa un patrón general por el cual la incidencia de la pobreza aumenta de manera independiente de los ciclos económicos. En las fases recesivas se incrementa el deterioro de los ingresos de los hogares, y en fases dinámicas éstos se recuperan, aunque más lentamente que en las de crisis. Más aún, las evidencias indican que cuando el PIB por habitante crece a tasas mayores de $3 \%$, aumenta la desigualdad, mientras que con crecimientos más bajos cercanos al estancamiento económico, se reduce la inequidad; en otras palabras, predomina la igualación hacia la pobreza (Filguera y Peri, 2004).

En el diagnóstico latinoamericano aún hay resistencia a aceptar que la reducción de la desigualdad y la pobreza son fundamentales para lograr mayor crecimiento económico; tampoco hay consenso en la forma de lograrlo y en qué magnitud. En ese sentido, además de las justificaciones propiamente éticas, se trata de que los logros en justicia distributiva e inclusión social intergeneracional formen parte central de la estrategia de desarrollo económico. En América Latina la riqueza se concentra históricamente en manos de las élites nacionales, regionales o locales; por ello en fases de alta creación de valor agregado no se obtienen grandes avances en la redistribución. En otras palabras, en estructuras sociales más homogéneas y con mayor integración de sus ciudadanos es posible tener mayor eficacia y eficiencia con esfuerzos más modestos de crecimiento y, por supuesto, con políticas explícitamente redistributivas. 
En el segundo argumento se ha puesto atención a los factores internos que explican - en algunos casos con gran peso- los resultados finales de los procesos de desarrollo o subdesarrollo, sin negar la tesis de que los factores externos asociados a la globalización con el liderazgo de grandes corporaciones trasnacionales y del capital financiero especulativo, sobredeterminen el desarrollo económico y social de los estados-nación, al obligarlos a aplicar medidas para abrir los mercados. ${ }^{4}$ Esa idea se relaciona con la capacidad de cada país, en un contexto internacional semejante, para diseñar e instrumentar políticas exitosas que logren reducir la desigualdad o atacar estructuralmente la pobreza. Un ejemplo de ello es la comparación entre naciones que han fracasado en la integración de políticas de fomento a la equidad en sus estrategias de desarrollo, como México (fase D de la curva de Kuznets), frente a otros que aún partiendo de condiciones de subdesarrollo han tenido éxito, como Vietnam (fase C) (PNUD, 2005).

\section{México}

En general, en México se observan las mismas tendencias predominantes que en América Latina en el largo plazo, aunque también tiene especificidades en relación con el crecimiento y la desigualdad. Sin tratar de encontrar la curva de Kuznets en el caso mexicano, se utiliza su planteamiento como punto de partida para explorar el comportamiento de algunas variables representativas a pesar de los problemas existentes en la información al respecto. ${ }^{5}$

\section{Desigualdad y crecimiento}

Existe una tendencia general de largo plazo en México entre 1950 y 2004, en la cual a mayor nivel del producto por habitante se reduce la desigualdad. Debemos anotar que la comprobación estadística es débil y que se observan etapas de mejoría y retroceso. La correlación obtenida entre el coeficiente de Gini y el PIB por habitante, para los datos disponibles entre 1950 a 2004, es de -0.43; es decir, es una correlación baja negativa. ${ }^{6}$

4 Al respecto Joseph Stiglitz dice que "ni la teoría ni la experiencia avalan la idea de que la apertura de los mercados a flujos de capital especulativo a corto plazo haga aumentar el crecimiento. Por el contrario, sí hay considerable experiencia y teoría para afirmar que incrementa la inestabilidad económica y que ésta contribuye a la inseguridad y la pobreza. Por consiguiente, dichas formas de liberalización de mercados de capital podrían, de algún modo, aumentar la 'globalización'. Pero lo que no hacen es mejorar el crecimiento y aunque éste se optimice ligeramente, el modo en que lo hace puede incrementar la pobreza, sobre todo en países que carecen de redes de seguridad social adecuadas" (PNUD, 2005:80).

5 Existen varios problemas en el estudio de largo plazo acerca de la distribución del ingreso y la pobreza derivados de la heterogeneidad de las cifras provenientes de las Encuestas de Ingreso Gasto de los Hogares, entre los cuales destacan el subreporte de ingresos y su incompatibilidad con las cuentas nacionales, así como diferencias de muestreo e instrumentación, mismos que se han intentado corregir con varios métodos (véase Hernández, 1999).

6 Cabe señalar que no se cuenta con Encuestas de Ingreso Gasto de los Hogares (ENIGH) para todo el periodo de 1950 a 2004, y los coeficientes de Gini disponibles fueron estimados por varios autores

\section{DeSarrollo}




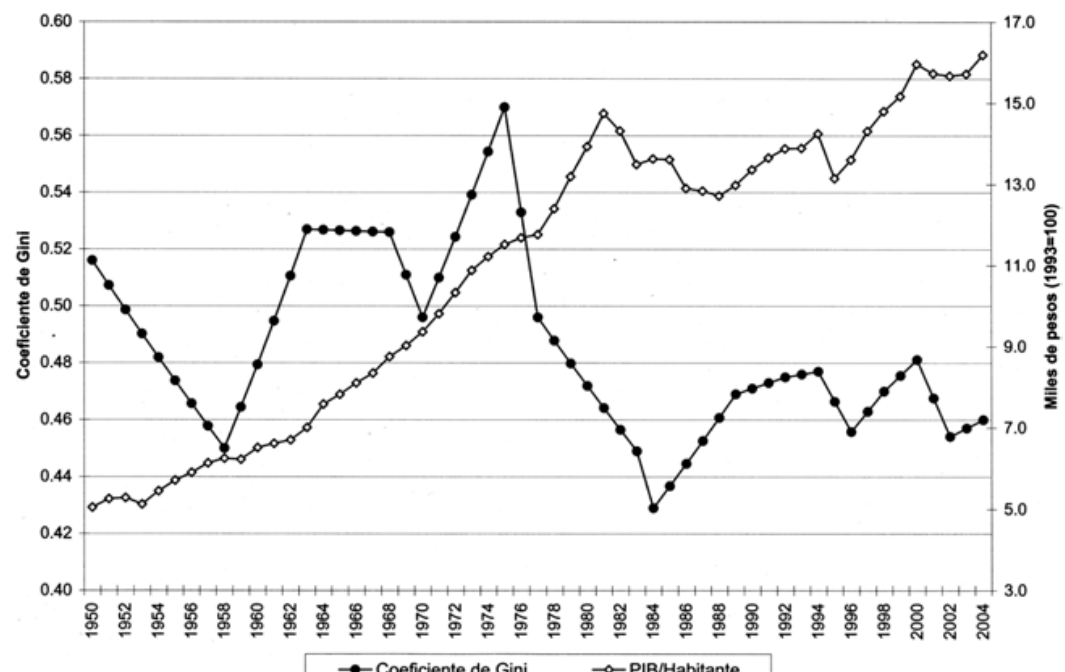

Gráfica 2. México. Coeficiente de Gini y PIB por habitante, 1950-2004.

La desigualdad en el ingreso se mantuvo en niveles altos en la etapa del modelo de sustitución de importaciones, de manera que el coeficiente de Gini aumentó hasta 0.57 en 1975, durante la fase denominada crecimiento con endeudamiento. No obstante, ese proceso se llevó a cabo con fuerte dinamismo de la economía, un aumento en el empleo formal remunerado y el salario real de los trabajadores. La desigualdad más baja fue en 1984 con un Gini de 0.43 , al inicio de la apertura comercial y el ajuste estructural. La crisis general y la caída del ingreso real, a partir de 1982 con la reducción en la concentración del ingreso, indican una etapa de igualación a la baja que afectó a amplios sectores de la clase media y se agudizó al repuntar todavía más hasta la crisis de 1994, cuando el coeficiente de Gini llegó a 0.48 , pero en condiciones de estancamiento. Y se mantuvo alrededor del 0.47 desde entonces hasta 2004, todavía en un nivel elevado, aunque con fluctuaciones y una tendencia a disminuir en los últimos años (véase Gráfica 2). ${ }^{7}$

En México, de acuerdo con el planteamiento de Kuznets, la fase A de crecimiento de la desigualdad operó durante el modelo de sustitución de importaciones con la transferencia de valor del campo a la ciudad y de la agricultura a la industria, que se extendió hasta 1975

a partir de las encuestas disponibles, lo que representa un problema de comparabilidad en series de largo plazo. En este trabajo se toman en cuenta las cifras existentes y se calculan los datos de los años faltantes de ese indicador mediante interpolación. En el periodo existen datos para 17 años, siendo la etapa de 1950 a 1989 el más irregular, mientras que entre 1992 y 2004 hay datos cada dos años.

7 Los datos en la disminución en la desigualdad y la pobreza durante esos años han sido cuestionados por su inconsistencia frente a otros indicadores como el bajo crecimiento económico y la mínima generación de empleo formal, así como diferencias en el diseño de la muestra y en los cuestionarios de las ENIGH de 2002 y 2004 que dificultan la comparación. Por otra parte, se mencionan como principales factores explicativos de dicha disminución las remesas provenientes de Estados Unidos, la contención de la inflación y el efecto de programas sociales (sobre todo PROCAMPO y Oportunidades).

\section{DeSarrollo}


sobre la base de un mayor endeudamiento público. La fase B habría llegado hasta 1984 con los procesos de urbanización e industrialización, y una reducción del coeficiente de Gini; en tanto que el PIB por habitante más alto se dio en 1981 (sólo hasta 1998 fue superado en términos reales). Posteriormente, se observa un comportamiento irregular en la desigualdad con fases de mejoría y deterioro, pero con una tendencia predominante orientada hacia la fase $\mathrm{D}$, caracterizada por una reducción en la creación de empleos en el sector industrial moderno del aparato productivo, pérdida de poder adquisitivo del salario, aumento de las actividades terciarias informales y deterioro en las condiciones de vida. La desigualdad se estabilizó, pero con alto grado de concentración de la riqueza, el cual ha significado un retroceso respecto de las condiciones observadas tres décadas atrás, y a pesar de un ingreso promedio creciente.

Hacia los años sesenta, la población urbana (residente en localidades de más de 2500 habitantes) superó a la rural en términos absolutos; para 2000 se multiplicó por cuatro y vino a representar las tres cuartas partes del total nacional (Gráfica 3).

Hacia 1970 se consolidó la transferencia de trabajadores del campo a la ciudad y la población económicamente activa (PEA) ocupada en el sector servicios superó a la PEA agrícola, en tanto que la PEA industrial superó a la PEA agrícola desde fines de los ochenta (Gráfica 4). La tendencia a la disminución de la desigualdad entre los años cincuenta y setenta respondería a un dinámico crecimiento de la economía y a la ocupación de los trabajadores en la industria. No obstante, el repunte en la concentración del ingreso y su estabilización en niveles altos desde los mediados de los ochenta estaría asociado a la interrupción del crecimiento económico, la contracción en la generación de empleos en el sector moderno, y a la consecuente incorporación de la población a actividades de servicios,

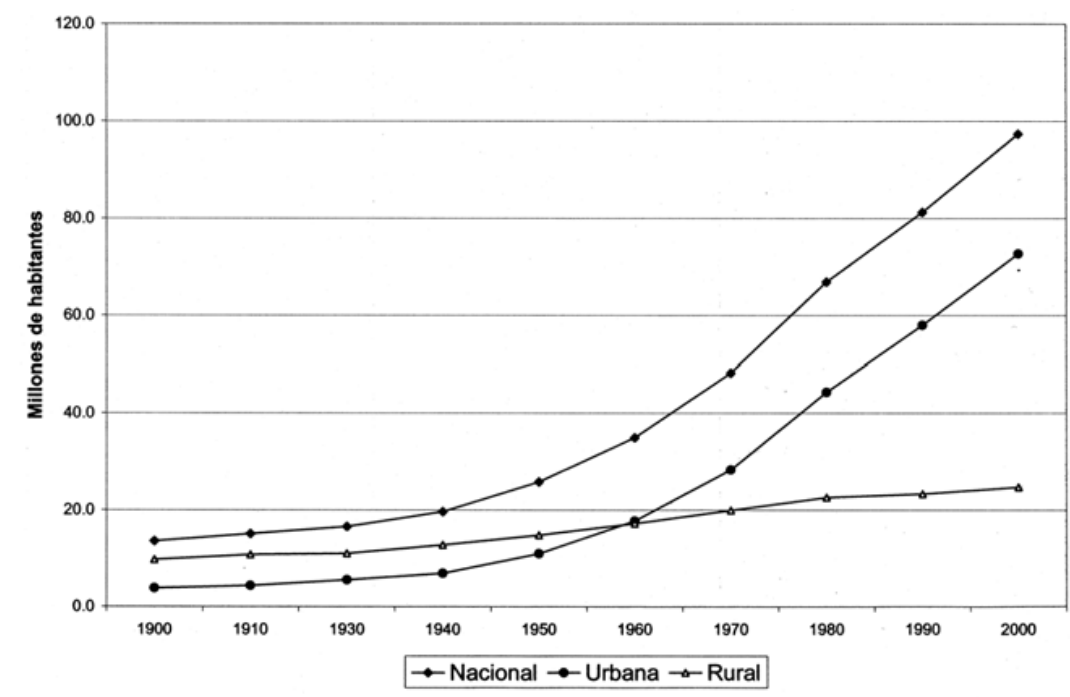

Gráfica 3. México, población censal urbana y rural, 1900-2000. 


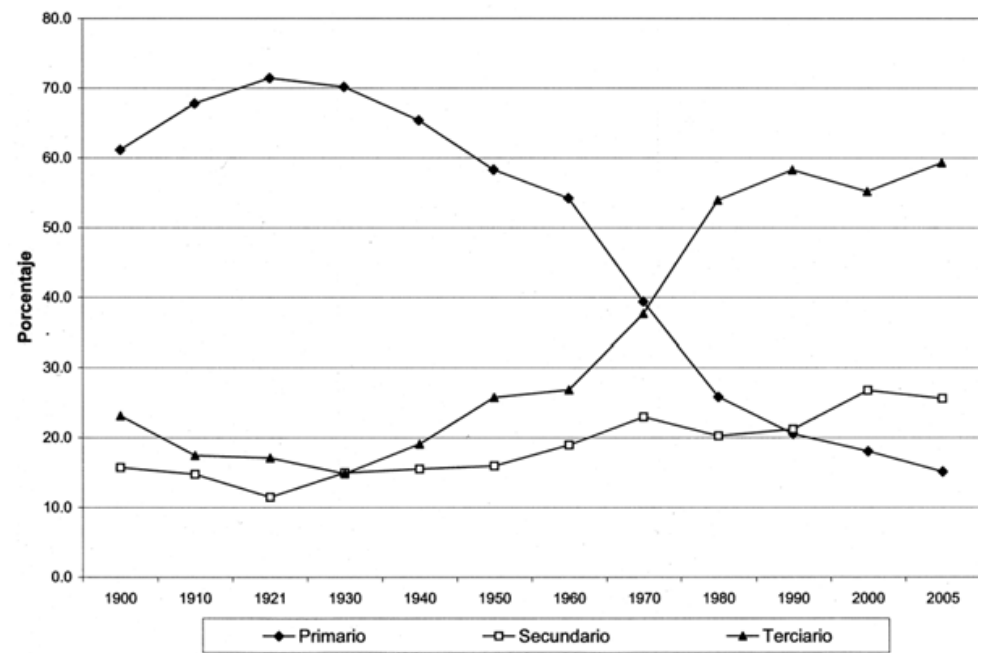

Gráfica 4. México, PEA por sector de actividad económica, 1900-2005.

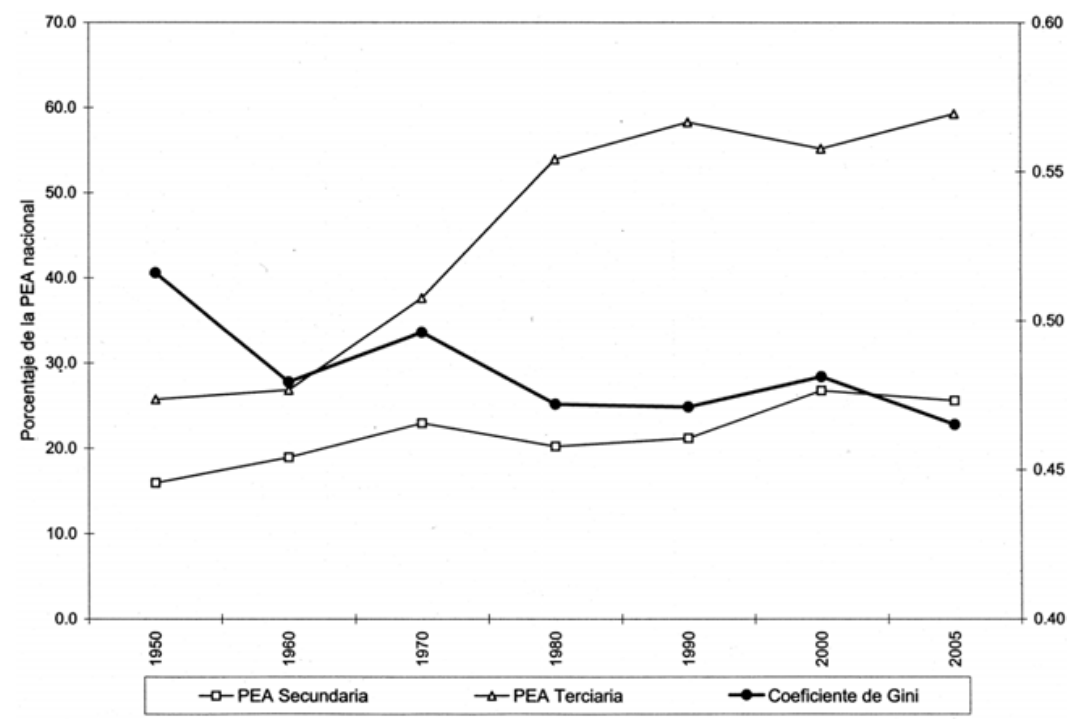

Gráfica 5. México, PEA por sectores secundario y terciario con coeficiente de Gini, 1900-2005.

en el marco de un modelo que amplificó la informalidad y la precariedad del trabajo, y generó en zonas urbanas y metropolitanas nuevos pobres expuestos a mayor vulnerabilidad (Gráfica 5).

A lo largo de todo el periodo, la PEA agrícola no mejoró su productividad media relativa (medida como relación directa entre PIB y PEA), mientras que la correspondiente a la PEA industrial, que llegó a ser cinco veces mayor que aquella en los sesenta, decayó 


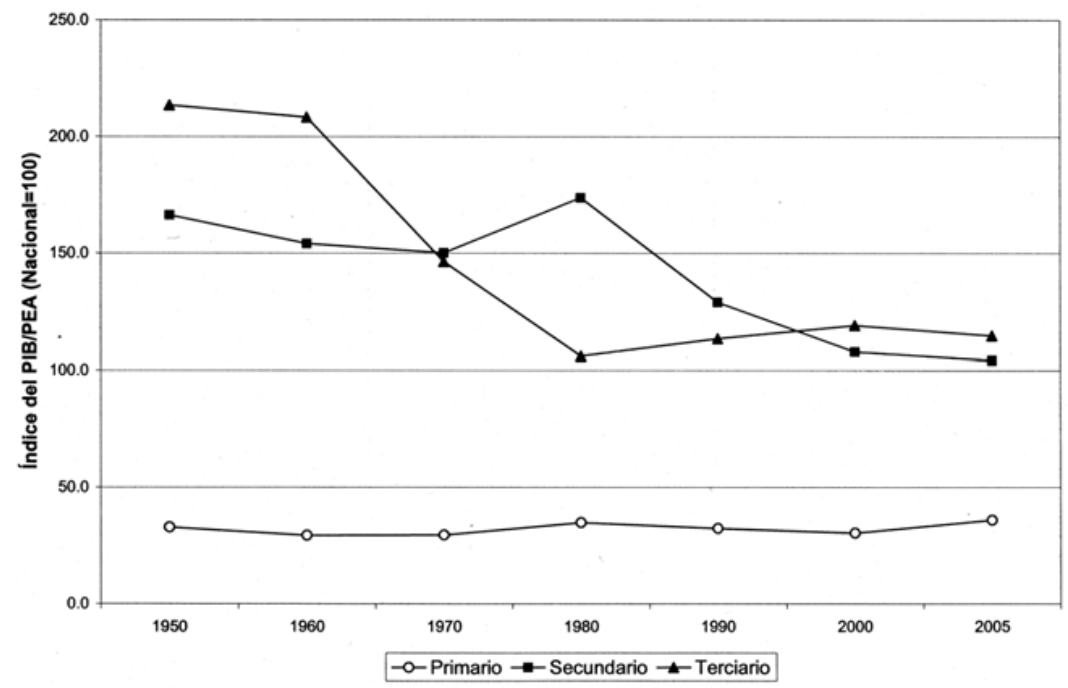

Gráfica 6. México, productividad media por sector de actividad económica, 1950-2005.

hasta representar sólo tres veces más en 2005. La productividad de servicios tuvo un comportamiento semejante pero de 7 a 3 veces. Esas tendencias indican la pérdida general de competitividad de los sectores no agrícolas de la economía (Gráfica 6).

Veamos ahora sus causas. La concentración del ingreso se mantiene muy alta. El modelo maquilador orientado a las exportaciones tiene características que inhiben el desarrollo económico, además de baja transferencia tecnológica y de habilidades, reducida integración con las cadenas nacionales y escasa generación de valor agregado. La renuncia del Estado a la conducción del mercado, que lleva, entre otras, a una baja recaudación fiscal, la reducción del gasto público y la debilidad de políticas como la industrial, la laboral y la de desarrollo urbano regional. Todo lo anterior, aunado a fenómenos como la corrupción y la inseguridad disminuyen en conjunto la competitividad (PNUD, 2005).

El nuevo modelo económico no se tradujo en mejoras en el bienestar social, sino en alto costo macrosocial, ya que se agudizaron algunos fenómenos como la desigualdad, el deterioro del salario real (que se considera una de las principales ventajas competitivas), el desempleo, la informalidad y la pobreza en el país. Se vivieron momentos de grave crisis sociopolítica y regional que pusieron en riesgo la estabilidad y la viabilidad de la democracia, debido al alejamiento del Estado de sus responsabilidades redistributivas. Con ello, a su vez, se debilitó la legitimidad del Estado.

\section{Crecimiento y pobreza}

Una hipótesis establece que la pobreza es un resultado del bajo crecimiento económico, ya que de ello depende la generación de empleo y el ingreso de la población, variables

\section{DeSarrollo}




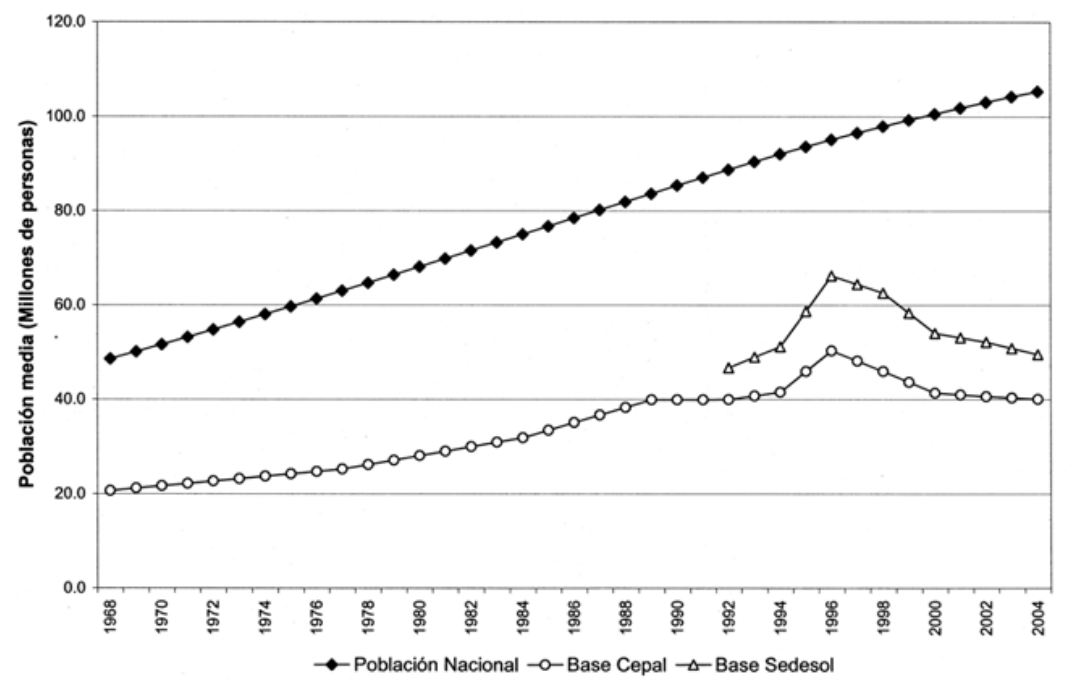

Gráfica 7. México, población media y estimaciones de pobres 1968-2004.

básicas en las condiciones de vida. Confirmarla requiere del manejo de diferentes conceptos, métodos de medición e información accesible sobre todos los aspectos en las series largas de la pobreza, de los cuales disponemos de manera irregular. ${ }^{8}$ No obstante, es posible una aproximación. Entre 1968 y 2004, la población media de México pasó de 47 a 105 millones de personas, mientras los que se encontraban en condiciones de pobreza, según la CEPAL, habrían aumentado de 21 a 40 millones de mexicanos. A su vez, de acuerdo con la SEDESOL, entre 1992 y 1996 (año cresta) los pobres habrían pasado de 47 a 66 millones de personas, para disminuir a 49 millones en 2004. En esa década, la crisis económica de 1995 provocó un dramático aumento en la pobreza total; sólo entre 1994 y 1996 creció en 14 millones de mexicanos, principalmente nuevos pobres víctimas de los "errores de diciembre", como expresión de una de las más graves crisis del capitalismo mexicano (Gráfica 7).

Una estimación amplia entre el PIB por habitante y el número total de pobres calculado por la CEPAL entre 1968 y 2004 indica una asociación positiva alta (0.76) entre ambas series; es decir, que a pesar de aumentar el nivel de ingreso promedio de la población también crece el número absoluto de personas en condiciones de pobreza (Gráfica 8). En ese sentido, la recuperación del ingreso real entre 1995 y 2000 se asocia con una disminución de la pobreza. Sin embargo, aunque el PIB por habitante se vuelve a estancar hacia 2003, los pobres aparentemente se reducen. Este patrón es inconsistente con las tendencias observadas y, de ser cierto, hay que buscar su explicación en factores como las remesas,

8 Existen datos estimados por varias instituciones o autores individuales para diferentes años pero de manera irregular. El caso de las estimaciones de CEPAL y SEDESOL, así como los años obtenidos mediante interpolación, se observan tendencias semejantes en los años en que coinciden, aunque no así en su magnitud. 


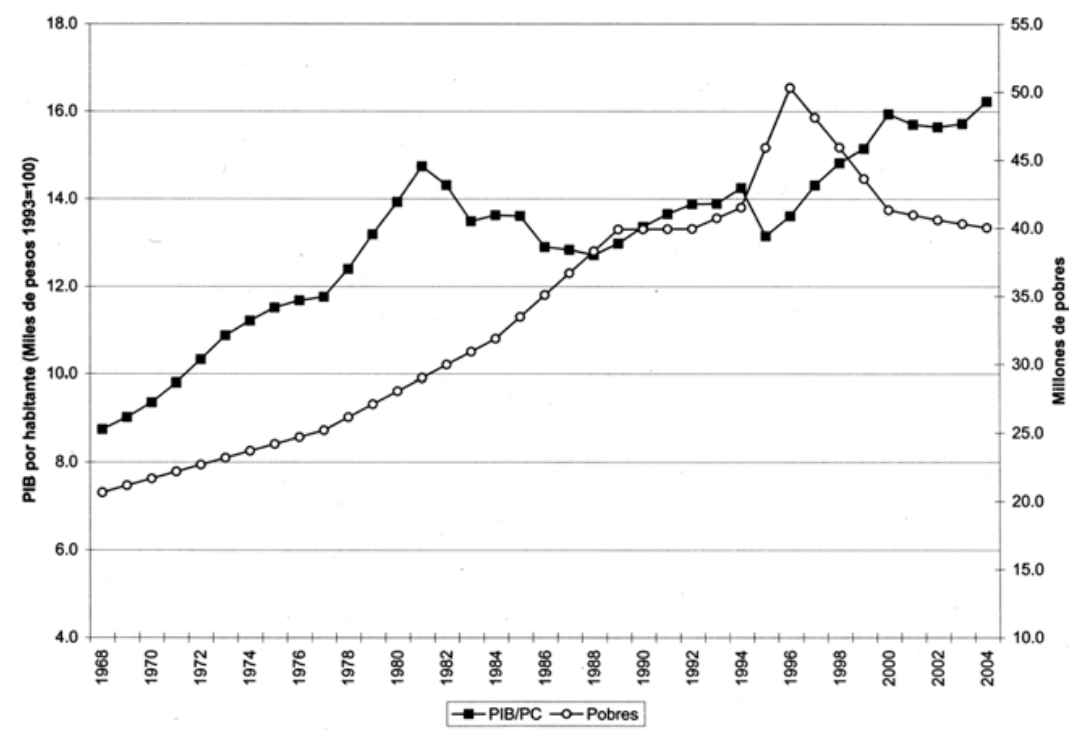

Gráfica 8. México, ingreso y pobres, 1968-2004.

que han aumentado hasta llegar a 20 mil millones de dólares en 2005 (de las cuales alrededor de $70 \%$ se destina al consumo doméstico), el control de la inflación, las actividades informales e ilegales (insuficientemente cuantificadas), así como los impactos directos generados por los programas sociales.

\section{Pobreza y gasto público}

Otra hipótesis afirma que las variaciones en la pobreza dependen de la intervención del Estado al corregir las fallas del mercado e introducir mecanismos redistributivos, y que el gasto público social es la principal variable (no la única) utilizada para ello. Asimismo, reconoce que no sólo se trata de la magnitud del gasto público como estabilizador social, sino de la eficacia y eficiencia con que éste se maneja — sobre todo aquél dirigido a la erradicación de la pobreza.

En México, el gasto social por persona se contrajo durante la década perdida de los ochenta y se recuperó ligeramente en los noventa. No obstante, la inequidad y la pobreza se han mantenido. En otros términos, no es suficiente canalizar mayores recursos presupuestales para superar esos rezagos sociales, es necesario evaluar la manera en que se gasta en el marco de una estructura social, institucional y de poder determinada.

Entre 1980 y 2004, el gasto público social tuvo un comportamiento procíclico como se manifiesta en su elevado coeficiente de correlación con respecto del PIB (0.92), lo cual confirma que ambas series aumentan y disminuyen paralelamente en etapas de crisis o de auge. Así, el gasto social se contrajo en vez de aumentar para enfrentar los costos sociales

\section{Desarrollo}




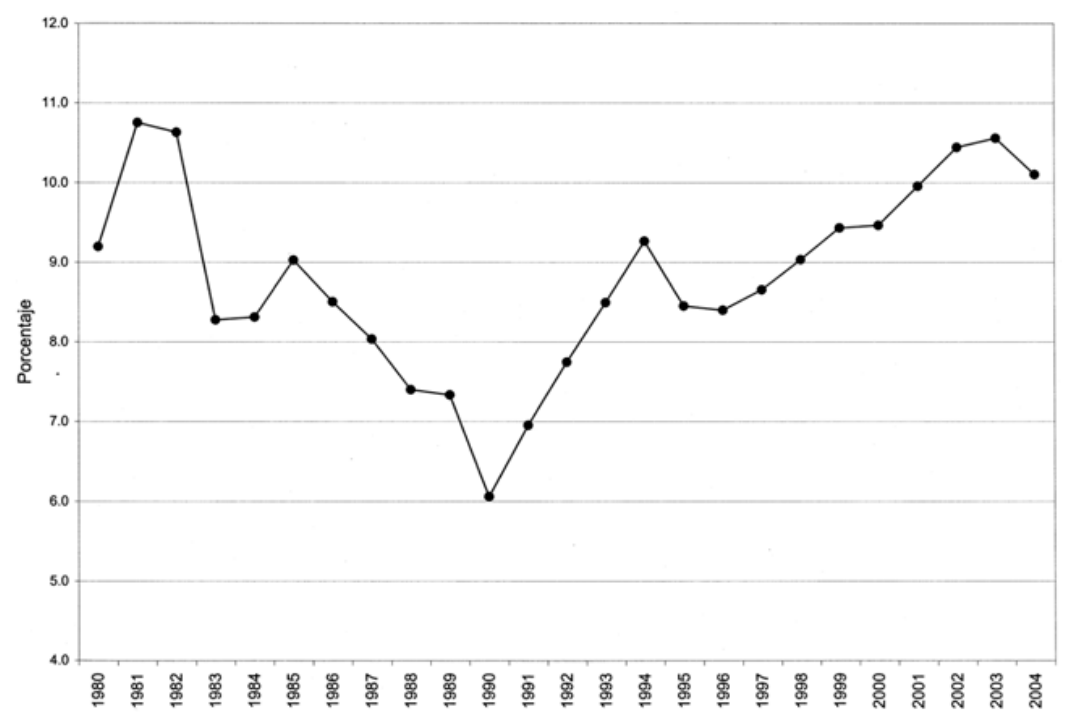

Gráfica 9. México, participación del gasto social en el PIB, 1980-2004.

de la década perdida y las crisis recurrentes, siendo 1990 el más bajo en relación con el PIB (sólo 6.1\%). Por ello se afirma que funcionó como la variable de ajuste para contener la crisis fiscal, con lo cual se redujo el salario indirecto y aumentó la pobreza de la población. 1994 fue otro punto de inflexión del gasto social, que no se recuperó hasta 1999, pero ya con el diseño de los programas enfocados a la pobreza extrema.

\section{Comparaciones internacionales}

La desigualdad en el patrón de distribución de la riqueza tiene gran importancia al analizar la relación entre ingresos promedio y niveles de pobreza entre países. En 2004, México se ubicó en el doceavo lugar entre 133 países, con un producto interno bruto total de 676 mil millones de dólares, debajo de naciones como Corea, India, Canadá y España; mientras que, según el ingreso nacional bruto por habitante, se colocó en el lugar 42 con 9590 dólares por persona, abajo de países como la Federación Rusa, Malasia, Chile y Croacia (World Bank, 2005). De acuerdo con el Índice de Desarrollo Humano (IDH), ${ }^{9}$ México se clasifica como un país de desarrollo humano alto y ocupó el lugar 53 entre 177 naciones en 2003. No obstante, si se ajusta sólo el componente del ingreso del IDH y se sustituye el ingreso promedio por habitante del país por el ingreso promedio de $20 \%$ de la población más pobre (indicador sensible a la desigualdad), y se mantienen los otros indicadores (de salud y educación) constantes, resulta que cae 55 lugares en la clasificación internacional

$9 \quad$ El IDH se calcula con indicadores de salud (esperanza de vida), conocimiento (escolaridad) e ingreso (PIB por habitante) (PNUD, 2005).

\section{Desararrollo}


y pasa del lugar 53 al 108. Tal es el impacto de la desigualdad en México. En otras palabras, es más grave la concentración de la riqueza que el lento crecimiento económico (PNUD, 2005).

\section{Las metas del milenio de la ONU}

En el marco de los Objetivos de Desarrollo del Milenio, una de las metas consiste en reducir a la mitad el porcentaje de población en pobreza extrema o indigencia entre 1990 y $2015 .{ }^{10}$ Para cumplir con dicha meta, el PIB por habitante promedio en el mundo debería crecer a una tasa mínima de $3.1 \%$ anual durante los siguientes once años. En América Latina la velocidad debería ser de por lo menos $2.9 \%$, en el supuesto de que la distribución actual de ingreso no tuviera mayores fluctuaciones. Si se logra mejorar tal distribución es posible potenciar el crecimiento económico para alcanzar esa meta. Por ejemplo, si el coeficiente de Gini se redujera 10\% (menor desigualdad), el producto por habitante necesario para lograr la misma meta para pobreza extrema se reduciría de 2.9 a $1.7 \%$ en promedio anual. Ahora bien, si se considera atacar la pobreza en general (no sólo la extrema), la tasa de crecimiento del PIB por habitante sería de $4.3 \%$ anual para esos próximos once años; pero si se corrige a la baja el coeficiente de Gini, ésta se reduciría a $2.5 \%$. Es decir, en las condiciones actuales, o se aumenta la velocidad del crecimiento económico o se reduce la concentración del ingreso, o ambas. Evidentemente, eso no excluye la aplicación de medidas asociadas indispensables tales como el diseño e instrumentación de políticas de inclusión social productiva para personas de más bajos ingresos, el aumento de los recursos públicos o mayor eficiencia en los programas sociales, entre otras.

En México, durante el periodo 1990-2000, la tasa de crecimiento del PIB por habitante fue de $1.5 \%$ y entre 2001 y 2005 se estima que sea de $0.7 \%$ anual; es decir, existe un rezago de $3.6 \%$ respecto de la tasa mínima necesaria, lo cual indica la magnitud del esfuerzo requerido solamente en función del objetivo de reducir la pobreza total.

\section{Comentario final}

La hipótesis de Kuznets ha ofrecido un modelo analítico sugerente para explicar con diferentes variables las relaciones entre crecimiento, desigualdad y pobreza. En México, a partir de ese enfoque, se observa en las últimas tres décadas un patrón de débil crecimiento económico y aumento de la desigualdad o de igualación a la baja en fases de recuperación, lo cual se explica por las condiciones históricas estructurales en la distribución social de la riqueza y el debilitamiento del papel del Estado como agente compensador de las

10 La pobreza extrema, según la onU, se estima con una línea de pobreza de menos de un dólar al día por persona, nivel que corresponde más a países de África y que subestima las necesidades monetarias en otras naciones tales como las de América Latina.

\section{DeSarrollo}


inequidades generadas en el mercado. También hay evidencia de que el mayor crecimiento y la menor desigualdad se retroalimentan, por lo que hay que promoverlas paralelamente.

Una estrategia central de los próximos gobiernos mexicanos será mantener, en un contexto mundial de mayor competencia, los equilibrios macroeconómicos básicos - condición necesaria pero insuficiente- y recuperar el dinamismo de la economía de manera complementaria a la aplicación de políticas redistributivas preferentemente de enfoque productivo para lograr ingresos y empleos permanentes y dignos, más allá de las medidas asistenciales tradicionales — de corto plazo— que seguirán siendo necesarias para el sector más vulnerable y empobrecido.

Las políticas de desarrollo con visión redistributiva para reducir las desigualdades sociales requieren de un nuevo pacto que haga posible combinar los esfuerzos y recursos de las esferas de la sociedad, la estatal y la mercantil en la promoción en varios frentes con mayor impacto social. Entre las medidas por considerar se pueden mencionar a manera de ejemplo las siguientes:

a) Capital humano. Destacan las políticas universales en los rubros de salud, educación y vivienda mediante los cuales los grupos más atrasados mejoran sus capacidades, con énfasis en los estados más rezagados como Chiapas, Guerrero, Oaxaca, Campeche, Yucatán, Veracruz, Hidalgo y Puebla, que reportan la mayor cantidad de municipios en condiciones de pobreza absoluta (con un alto índice de riesgo nutricional y mortalidad); será necesario también revisar el diseño de algunos programas sociales como PROCAMPO y Oportunidades, para hacerlos más eficientes en la lucha contra la pobreza, mediante estrategias de apoyo al patrimonio familiar (vivienda con servicios básicos) y a proyectos productivos sustentados con modalidades de capital social, pero alejados de prácticas clientelares o corporativas.

b) Capital físico y de inversión. El impulso a programas específicos como los dirigidos a las micro, pequeñas y medianas empresas, y a la infraestructura básica requerida por comunidades de difícil acceso (carreteras y caminos rurales), para mejorar los términos de intercambio entre el sector agrícola y el resto de la economía, lo que implica el aumento de los ingresos fiscales y del gasto público, así como mayor y mejor coordinación intergubernamental.

c) Capital natural. El cual debe tomar en cuenta el cuidado de ecosistemas a lo largo y ancho del país para garantizar su sustentabilidad, su aprovechamiento en proyectos, como los ecoturísticos, y aun el apoyo estatal para garantizar ingresos por cuidados y servicios ambientales a los propietarios o usufructuarios de los recursos naturales; se debe igualmente avanzar en la asignación de responsabilidades sociales, sectoriales y territoriales por su uso.

\section{$\underline{\text { DeSarar prollo }}$}


d) Desarrollo regional. Debe promoverse con el objetivo de reducir las brechas entre los distintos niveles territoriales del país, con adaptación a procesos globales, con preferencia de modelos endógenos para lograr el éxito, aprovechando las ventajas de los procesos de descentralización, así como con el fortalecimiento de los gobiernos y la creciente participación de las sociedades locales en el diseño y evaluación de políticas y programas de desarrollo.

Bibliografía

Aguilera Verduzco, Manuel, Crecimiento económico y distribución del ingreso, Balance teórico y evidencia empírica, México, Facultad de Economía-ENEP Acatlán, UNAM, 1998.

Asuad, Norman, "La estrategia del cambio: ¿modernización sectorial o desarrollo regional y territorial?, en Javier Delgadillo (coordinador), Planeación territorial, políticas públicas y desarrollo regional en México, México, CRIM, DGAPA, UNAM, 2004, pp. 137-184.

Bowman, Kirk S., "Should the Kuznets effect relied on to induce equalizing growth: evidence from Post-1950 development", en World Development, vol. 25, núm. 1, 1997, pp. 127-143.

Bruno, Michael, Martín Ravallion and Lyn Squire, "Equity and growth in developing countries", en Policy Research Working Paper, 1563, The World Bank, enero, 1996.

CEPAL, Una década de luces y sombras: América Latina y el Caribe en los años noventa, AlfaomegaCEPAL, Santiago, Chile, 2001.

Cortés, Fernando y Rosamaría Ruvalcaba, Técnicas estadísticas para el estudio de la desigualdad social, México, El Colegio de México-Flacso, 1984.

Dasgupta, Partha, "La riqueza perdida de las naciones", en Project Syndicate, 2005, www. project-syndicate.org.

Filguera, Carlos y Andrés Peri, América Latina: los rostros de la pobreza y sus causas determinantes,
Serie Población y Desarrollo, núm, 54, Santiago de Chile, CEPAL, 2004.

Galor, Oded y Daniel Tsiddon, "Income distribution and growth: The Kuznets hypothesis revisited", en Economica, New Series, vol. 63, núm. 250, 1996, pp. 103-117.

Hernández, Enrique, "Evolución de la distribución del ingreso en los hogares (1963-1989)", en Julio Boltvinik y Enrique Hernández, Pobreza y distribución del ingreso en México, México, Siglo XxI, 1999, pp. 154-190.

Kanbur, Rabi, "Income distribution and development", en Anthony B. Atkinson y Francois Bourguignon (editores), Handbook of Income Distribution, Amsterdam, North Holland, 2000.

Kuznets, Simón, "Economic growth and income inequality", en The American Economic Review, vol. 45, núm. 1, marzo, 1955, p. 1-28.

-, "Aplicación de las estimaciones de renta nacional en el análisis y política de crecimiento económico", en varios, El ingreso y la riqueza, México, FCE, 1963, pp. 7-23.

Mkandawire, Thandika, Social policy in developing context, Suiza, UNRISD, Paper number 7, 2001.

PNUD, Informe sobre el desarrollo humano 2005. La cooperación internacional ante una encrucijada. Ayuda al desarrollo, comercio y seguridad en un mundo desigual, Nueva York, Mundi-Prensa, 2005.

World Bank, World Development Report 2006, Equity and development, Washington, D.C, 2005. 